\title{
Molecular-based detection of the gastrointestinal pathogen Campylobacter ureolyticus in unpasteurized milk samples from two cattle farms in Ireland
}

\author{
Monika Koziel ${ }^{1}$, Brigid Lucey ${ }^{1,2^{*}}$, Susan Bullman ${ }^{1}$, Gerard D Corcoran ${ }^{2}$ and Roy D Sleator ${ }^{1}$
}

\begin{abstract}
Campylobacter jejuni and coli are collectively regarded as the most prevalent cause of bacterial foodborne illness worldwide. An emerging species, Campylobacter ureolyticus has recently been detected in patients with gastroenteritis, however, the source of this organism has, until now, remained unclear. Herein, we describe the molecular-based detection of this pathogen in bovine faeces (1/20) and unpasteurized milk (6/47) but not in poultry (chicken wings and caeca). This is, to the best of our knowledge, the first report of the presence of this potential gastrointestinal pathogen in an animal source, possibly suggesting a route for its transmission to humans.
\end{abstract}

Keywords: Campylobacter, Emerging pathogen, Food chain, Reservoir, Dairy

\section{Correspondence}

Human campylobacterosis is generally regarded as a zoonosis with numerous reservoirs in the natural environment [1]. While Campylobacter spp. rarely cause disease in livestock, foodstuffs originating from such animals appear to be the most common source of infection for humans.

Despite the fact that there are 25 species belonging to the Campylobacter genus [2], the primary focus of the food safety and protection agencies to date, in conjunction with clinical laboratories, has been the detection of thermophilic Campylobacter species associated with human diseases [3,4]. However, advances in molecular detection systems continue to highlight the fact that routine Campylobacter culture methods, employed by the majority of clinical laboratories, are incapable of detecting the fastidious and non-thermophilic Campylobacter spp [5]. Recent work in our laboratory has focused on the identification and characterisation of these atypical species of potential clinical importance $[3,6]$. Although failing to grow in routine culture, species specific

\footnotetext{
* Correspondence: Brigid.Lucey@cit.ie

'Department of Biological Sciences, Cork Institute of Technology, Rossa

Avenue, Bishopstown, Cork, Ireland

${ }^{2}$ Department of Microbiology, Cork University Hospital, Wilton, Cork, Ireland
}

PCR following a multiplex PCR based detection system (EntericBio, Serosep Ltd. Limerick, Ireland) identified $C$. ureolyticus in $23.8 \%$ of 349 previously genus-positive samples [6], making $C$. ureolyticus the second most common Campylobacter species (after C. jejuni) detected in faecal samples of patients presenting with gastroenteritis in Southern Ireland. Moreover, this species has also been detected and isolated from a number of patients presenting with Crohn's disease [7] and Ulcerative Colitis [8]; raising further questions as to its potential role as a significant human pathogen. Importantly, C. ureolyticus has been shown to be capable of attaching and translocating through the intestinal epithelia, inducing cellular damage and microvillus degradation [9].

The source of $C$. ureolyticus has, until now, remained unknown. Herein, we report for the first time the presence of this bacterium in cow's milk, which goes some way to answering the important question as to where this emerging gastrointestinal pathogen might be coming from.

A total of 40 chicken caeca and 20 chicken wings were obtained directly from an Irish broiler processing farm between 26 January and 14 February, 2012. Bovine samples consisting of: 47 unpasteurised milk samples, 20 midstream urine samples and 20 faecal samples were 
Table 1 Primer sequences forward (F) and reverse (R) used in this study

\begin{tabular}{llll}
\hline Primer & Primer Sequence $\left(\mathbf{5}^{\prime} \rightarrow \mathbf{3}^{\prime}\right)$ & Product (bp) & Ref. \\
\hline CU-HSP60 F & GAA GTA AAA AGA GGA ATG GAT AAA GAA GC & 429 & {$[6]$} \\
\hline CU-HSP60 R & CTT CAC CTT CAA TAT CCT CAG CAA TAA TTA AAA GA & 196 & \\
\hline Pgi F & AAA CAC CTT CAC GAC TTA CCG & \\
\hline Pgi $R$ & CCA ACT CGA ACA GTA GGG ACA & \\
\hline
\end{tabular}

also collected from individual cows between 19 April and 19 May 2012, from two Southern Irish farms, located approximately $80 \mathrm{~km}$ apart. Bacterial DNA was extracted using QIAamp DNA Stool Mini Kit (QIAGEN) for both chicken sample types and bovine faeces and QIAamp DNA Mini Kit (QIAGEN) for bovine urine and milk samples. Each extraction run included an extraction control - a sample matrix spiked with the $C$. ureolyticus DSM 20703 strain. The presence of C. ureolyticus in each sample was investigated using PCR with a specific primer set targeting the hsp60 gene of C. ureolyticus [6] Figure 1. An internal amplification control (IAC), designed using DNA from $N$. gonorrheae and N. gonorrheae-specific Pgi primer [10], was included in each reaction to assess potential PCR inhibition. The sequences of all primers used in the study are outlined in Table 1. PCR was performed using HotStarTaq DNA Polymerase (QIAGEN) with the following cycle conditions: initial denaturation for $15 \mathrm{~min}$ at $95^{\circ} \mathrm{C}$, followed by 35 cycles of denaturation at $95^{\circ} \mathrm{C}$ for $30 \mathrm{~s}$, annealing at $58^{\circ} \mathrm{C}$ for $1 \mathrm{~min}$, extension at $72^{\circ} \mathrm{C}$ for 1 min and final extension at $72^{\circ} \mathrm{C}$ for $10 \mathrm{~min}$.

Amplicons were visualised by electrophoresis on agarose gels and the results were interpreted on the basis of presence/absence of a band for C. ureolyticus positive samples and an IAC amplification for C. ureolyticus negative samples.

We have investigated a total of 60 chicken samples as potential reservoirs of $C$. ureolyticus. However, none gave the amplicon of the predicted size and as such were deemed negative for the presence of $C$. ureolyticus. The
IAC was positive in all reactions. The fact that all of the chicken samples screened throughout the period of the study were negative for C. ureolyticus-specific PCR suggested that, unlike the thermophilic $C$. jejuni, chickens might not be the main reservoir for $C$. ureolyticus.

Failure to detect $C$. ureolyticus in chickens prompted us to investigate cattle, which have also been reported as a source of Campylobacter spp [11]. A total of 87 bovine samples were screened and while no $C$. ureolyticus was detected in urine samples; one faecal sample and six milk samples were positive for $C$. ureolyticus (Additional file 1). These positive samples were collected from six different cows across two geographically separate herds. To ensure the validity of the positive results, the PCR amplicons were sequenced using previously described forward and reverse $h s p 60$ primers. The sequences were analysed by BLAST using the NCBI database and their identity was confirmed as the C. ureolyticus hsp 60 gene.

Data reported for the prevalence of $C$. ureolyticus in patients with gastroenteritis in Ireland suggest a seasonal distribution of the organism, with the majority of cases reported in early spring, particularly during the month of March [3]. The detection of C. ureolyticus in bovine samples during this period suggests the need for a comprehensive prospective study to determine the incidence of this organism in cattle throughout the calendar year for a comparison with the seasonal distribution that has already been observed among patients with gastroenteritis [3]. Furthermore, comparison of MLST profiles of those strains isolated from the cattle and patients with gastrointestinal (GI) illness will significantly aid in

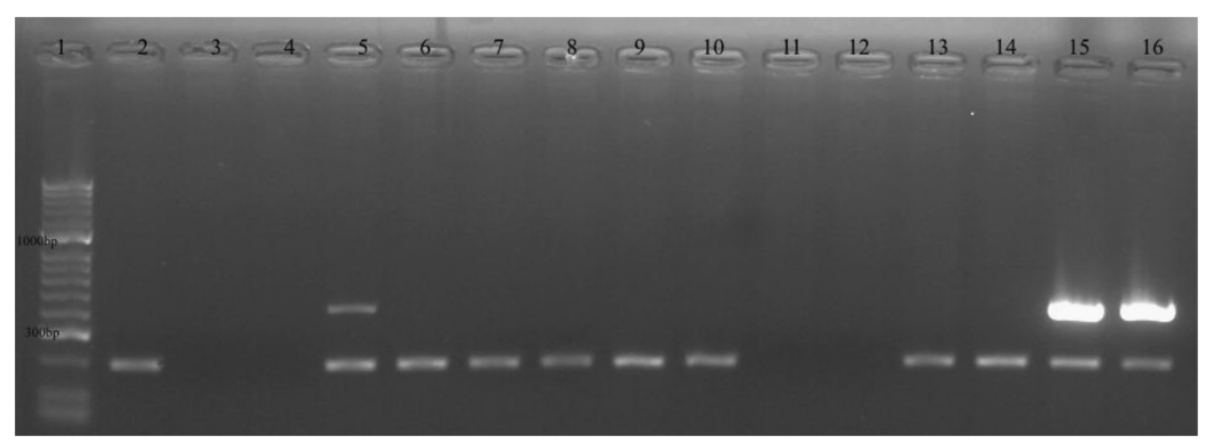

Figure 1 1.5\% agarose gel with PCR amplicons. An example of a C. ureolyticus PCR-positive result in milk sample with a product of 429bp (Lane 5). Lane 1 100bp molecular marker; Lane 2 negative (no template) control; Lane 3-14 Milk samples; Lane 15 Extraction positive control; Lane 16 PCR positive control. 
determining if cow's milk is in fact the true source of C. ureolyticus in patients with GI illness and if $C$. ureolyticus, like many of the other Campylobacter species, can be added to the growing list of zoonotic pathogens.

While more extensive studies are needed to investigate the true prevalence of the $C$. ureolyticus in cattle; the current study is, to the best of our knowledge, the first report of the presence of this bacterium in animal samples, providing a clue to a potential source of $C$. ureolyticus in the food chain.

\section{Additional file}

Additional file 1: Details of the samples tested in this study and their results.

\section{Competing interests}

The authors declare that they have no competing interests.

\section{Acknowledgements}

The authors acknowledge the financial assistance of Serosep Ltd, Ireland. MK and SB are recipients of PhD fellowships from the Irish Research Council RS/2011/264 and RS/2009/1670 respectively. RDS is an ESCMID Research Fellow. We thank Vincent Carton and Lynn Farrelly of Manor Farm Chickens, and Helena Stack and Philip Murphy for the provision of animal samples.

Received: 18 October 2012 Accepted: 4 November 2012

Published: 14 November 2012

\section{References}

1. Moore JE, Corcoran D, Dooley JS, Fanning S, Lucey B, Matsuda M, et al: Campylobacter. Vet Res 2005, 36:351-382.

2. Man SM: The clinical importance of emerging Campylobacter species. Nat Rev Gastroenterol Hepatol 2011, 8:669-685.

3. Bullman S, Corcoran D, O'Leary J, O'Hare D, Lucey B, Sleator RD: Emerging dynamics of human campylobacteriosis in Southern Ireland. FEMS Immunol Med Microbiol 2011, 63:248-253.

4. Whyte P, McGill K, Kelly L, Cowley D, Fanning S, Collins D, et al: A Comparative Study of Thermophilic Campylobacter Isolates of Clinical, Food and Pet Origin. Safe food, Ireland: Food Safety Promotions Board; 2006.

5. Bullman S, Lucey B, Sleator RD: Molecular diagnostics: the changing culture of medical microbiology. Bioeng Bugs 2012, 3:1-7.

6. Bullman S, Corcoran D, O'Leary J, Lucey B, Byrne D, Sleator RD: Campylobacter ureolyticus: an emerging gastrointestinal pathogen? FEMS Immunol Med Microbiol 2011, 61:228-230.

7. Zhang L, Man SM, Day AS, Leach ST, Lemberg DA, Dutt S, et al: Detection and isolation of Campylobacter species other than C. jejuni from children with Crohn's disease. J Clin Microbiol 2009, 47:453-455.

8. Mukhopadhy I, Thomson JM, Hansen R, Berry SH, El-Omar EM, Hold GL: Detection of Campylobacter concisus and other Campylobacter species in colonic biopsies from adults with ulcerative colitis. PLoS One 2011, 6:e21490.

9. Burgos-Portugal JA, Kaakoush NO, Raftery MJ, Mitchell HM: Pathogenic potential of Campylobacter ureolyticus. Infect Immun 2012, 80:883-890.

10. O'Callaghan I, Corcoran D, Lucey B: Design of a multiplex PCR assay for the simultaneous detection and confirmation of Neisseria gonorrhoeae. J Clin Pathol 2010, 63:431-433.

11. Wilson DJ, Gabriel E, Leatherbarrow AJ, Cheesbrough J, Gee S, Bolton E, et al: Tracing the source of campylobacteriosis. PLoS Genet 2008, 4:e1000203.

doi:10.1186/1757-4749-4-14

Cite this article as: Koziel et al:: Molecular-based detection of the gastrointestinal pathogen Campylobacter ureolyticus in unpasteurized milk samples from two cattle farms in Ireland. Gut Pathogens 2012 4:14.

\section{Submit your next manuscript to BioMed Central and take full advantage of:}

- Convenient online submission

- Thorough peer review

- No space constraints or color figure charges

- Immediate publication on acceptance

- Inclusion in PubMed, CAS, Scopus and Google Scholar

- Research which is freely available for redistribution 